THEORÍA

REVISTA DEL COLEGIO DE FILOSOFÍA
NÚMERO 41 | DICIEMBRE 2021 - MAYO 2022 | 169-183

DOI: $10.22201 /$ ffyl.16656415p.2021.41.1567

Recibido: 1-08-2021 | Aceptado: 18-10-2021

\title{
EL MUNDO EXPRESADO INTERSUBJETIVAMENTE: UN PUENTE ENTRE NISHIDA Y HUSSERL
}

\author{
An Intersubjective World: A Link Between Nishida and Husserl ${ }^{1}$
}

\author{
Daniel Salvador Alvarado Grecco \\ Universidad Nacional Autónoma de México | México \\ Contacto:d_grecco96@comunidad.unam.mx
}

\section{Resumen}

En lo que sigue me propongo trazar un vínculo entre los planteamientos de Nishida y Husserl en lo que respecta la intersubjetividad. Si bien hay diferencias importantes entre estos autores, encuentro que coinciden en el tratamiento del mundo expresado intersubjetivamente, es decir, aprehendido por una multiplicidad de individuos. Para demostrar lo anterior, me centraré en los aspectos que hacen casi imposible cualquier tentativa de vincular los planteamientos de los dos, a saber, el hecho de que Nishida y Husserl parecen ir en distintas direcciones debido al uso de conceptos como 'experiencia pura, 'reducción fenomenológica’ y epojé. Sugiero, sin embargo, que estas diferencias deben ser examinadas a partir de las observaciones de "The Logic of Topos" - Nishida-y Meditaciones cartesianas - Husserl-. A mi parecer, la coincidencia ha de ser encontrada en estos textos, ya que los dos ofrecen la idea de un mundo expresado a través de la aprehensión del mundo del individuo. Mis conclusiones intentarán ampliar el alcance de este artículo al sugerir algunos vínculos entre Nishida y la obra fenomenológica de Jacques Derrida, cuyo trabajo intentó poner la cuestión de la alteridad al centro de la fenomenología.

Palabras clave: alteridad, intersubjetividad, reducción fenomenológica, experiencia pura, lógica del topos, conciencia

\begin{abstract}
In this article I intend to establish a link between Nishida and Husserl in regard to their ideas about intersubjectivity. Although there are significant differences concerning these philosophers' ideas, I think that they agree on conceiving the world as intersubjectively expressed, that is, as apprehended by a multiplicity of individuals. To demonstrate this, I will focus on those aspects that make any attempt to link these philosophers close to impossible, namely, the fact that Nishida and Husserl seem to follow two different directions because of the concepts 'pure experience,' phenomenological reduction,' and époché. However, I argue that these differences should be examined under the observations of "The Logic of Topos"-Nishida-and Cartesian MeditationsHusserl. In my opinion, it is in these two texts that any coincidence is to be found since they provide the idea of a world that's expressed through the individual's apprehension of the world. My conclusions will try to expand the scope of this article by suggesting some connections between Nishida and the phenomenological work of Jacques Derrida, whose philosophy attempted to place the question of alterity at the core of phenomenology.
\end{abstract}

Keywords: alterity, intersubjectivity, phenomenological reduction, pure experience, logic of topos, consciousness

1 Este trabajo se hizo como parte del proyecto PIFFYL 02_006_2019 "Ontologías intersticiales" de la Facultad de Filosofía y Letras de la Universidad Nacional Autónoma de México (unAM). 
El otro es reflejo de mímismo y, sin embargo, no es estrictamente reflejo; es un analogon de mí mismo y, sin embargo, no es un analogon en el sentido habitual.

Edmund Husserl, Meditaciones Cartesianas

A self must include an absolute other in itself, an absolute negation in itself. It is not that a self becomes other and other becomes the self through a medium. It is rather that the self becomes the other through the bottom of itself.

Nishida, "I and Thou”

\section{Introducción}

$\mathrm{L}$

a intersubjetividad es uno de los puntos obligados en toda filosofía que se reivindique como intersticial. En efecto, su abordaje siempre responde al hecho de que el mundo, en cuanto compartido por otros sujetos, es irreducible a mi yo; que la alteridad siempre se asoma en lo que considero más propio e íntimo. Es en este aspecto en el que creo encontrar una coincidencia entre Kitarō Nishida y Edmund Husserl, pensadores que comparten ciertas inquietudes, como la fidelidad a la experiencia, pero también muchas diferencias importantes. No debe sorprender, entonces, que haya esfuerzos por aliar ambos pensamientos. Sobresale así Shigeru Taguchi, filósofo que traza la coincidencia entre Nishida y Husserl a partir de una concepción no contextual de la conciencia (Taguchi, 2019: 99). De acuerdo con Taguchi, dado que Husserl y Nishida insistieron en la irreductibilidad entre la conciencia y su objetivación, cualquier coincidencia entre estos dos autores tiene que ser encontrada en la compenetración originaria y no espacial del Yo y el Otro. ${ }^{2}$

Aunque plausible y rica en fuentes, creo que la perspectiva de Taguchi deja de lado un aspecto importante en el tratamiento que tanto Nishida como Husserl hacen de la intersubjetividad, a saber, que la objetividad del mundo se compone a partir de las distintas aprehensiones de los individuos. Además, las descripciones de Husserl en la quinta meditación cartesiana complican lo anterior en un aspecto, a saber, la inclusión del cuerpo y, por ende, de la espacialidad. Cierto, hay un Yo-originario anterior a la aprehensión del yo personal y corporal (Taguchi, 2019: 103), pero, tal y como desarrollaré más adelante, la aparición del otro en mi campo visual implica una constitución

2 Con irreductibilidad me refiero al hecho de que la conciencia excede su determinación como conciencia que pertenece a un yo en particular. 
que se funda, en primer lugar, en la semejanza entre nuestros cuerpos y, en segundo, en la referencia a un 'allî.

En las páginas que siguen intentaré desarrollar esta intuición. En primer lugar, prestaré atención a los conceptos que parecen complicar cualquier tentativa de coincidencia entre Nishida y Husserl. Es innegable que ambas perspectivas van en sentidos opuestos, tal y como puede testimoniar el contraste entre la experiencia pura, la reducción y la epojé fenomenológica. Finalmente, intentaré tender un puente entre los planteamientos de ambos a partir de las observaciones de "The Logic of Topos" (Nishida, 1986) y Meditaciones cartesianas (Husserl, 1973), textos en donde los dos autores abordaron cuestión del mundo como aprehendido por varios individuos.

\section{La unidad que subyace a la experiencia}

En Invitación a la filosofía japonesa, Bernard Stevens (2008) ofrece una pista valiosa para entender la divergencia entre los planteamientos de Nishida y Husserl. Con la experiencia pura, Nishida buscaba realizar una descripción más exacta de la vida de la conciencia, razón por la cual se sintió atraído por el imperativo fenomenológico "hay que retornar a las cosas mismas" (Stevens, 2008: 151). Sin embargo, ¿la escisión entre nóesis y nóema, acto de conciencia y sentido intencionado, satisfizo la inquietud de Nishida? Todo lo contrario; al hacer de dicha distinción "el hilo conductor de la fenomenología trascendental", Husserl incurría, a los ojos de Nishida, en los mismos problemas de filosofías precedentes, a saber, romper la inmediatez originaria de la experiencia mediante categorías que le son totalmente ajenas (Nishida, 1995: 41). ${ }^{3} \mathrm{Si}$ a dicha objeción se suma el dejo idealista de Ideas I que Nishida encontró en Husserl, ${ }^{4}$ se puede preguntar con toda legitimidad si no existe un profundo desacuerdo entre ambos pensadores. A continuación, me propongo mostrar uno de los puntos que me parecen irreconciliables entre Husserl y Nishida: el modo en el que los dos se hicieron de una vía para indagar sobre la conciencia.

3 Otro problema que surge de lo anterior está en la objetivación del proceder reflexivo: la fenomenología no sólo rompe, retomando a Maraldo (2017), "la conciencia que es consciente ahora" [consciousness that is now conscious]" (308); también hace de la conciencia un objeto a partir de su descripción.

4 El “dejo idealista” puede entenderse a partir de un elemento problemático de Ideas I: la distinción de la conciencia como un absoluto frente a la relatividad y contingencia del mundo. A dicha idea subyace el modo de dación de la cosa trascendente frente al de la vivencia. Mientras que los objetos físicos se dan por escorzos, la vivencia se da de manera absoluta (Husserl, 2013: 165-16 [85]; en adelante Ideas I). 
Una de las ideas que más se repiten en Indagación del bien, primera obra de Nishida, es la insistencia en la simplicidad de la experiencia. Con ello no se afirma su indiferenciación, como si a Nishida se le hubiera olvidado el entramado de estados y actos que componen la experiencia; antes bien, se afirman como sus características originales la unidad y la inmediatez, detalles que la psicología y la fenomenología habían perdido de vista debido a la naturaleza reflexiva de sus acercamientos. La experiencia pura, hilo conductor de Indagación, incluye toda vivencia mental, ya sean sensaciones o percepciones, actos actuales o recuerdos. Lo que se acentúa aquí es el hecho de que estas vivencias se dan en el momento presente; poco importa entonces su índole o la manera en que se integran en el flujo total de la conciencia. Sobre esta base pueden entenderse las observaciones de Nishida respecto al recuerdo: aun cuando no aprehendamos directamente lo que la memoria nos presenta, el recuerdo nos afecta en el momento presente (Nishida, 1995: 43). Aunado a lo anterior, la escisión entre sujeto y objeto, tan característica de las filosofías de la representación, es diluida por Nishida al precisar que la conciencia y sus objetos, sea cual sea su índole, coexisten en un presente que no admite dicotomías. La unidad no implica, sin embargo, la anulación del yo o, dicho en términos fenomenológicos, la perspectiva de la experiencia desde la primera persona. Tal y como indica John C. Maraldo (2017b), los desplazamientos conceptuales de Nishida en la Indagación tienen el propósito de elaborar "una subjetividad sin sujeto", una idea de experiencia en donde la conciencia deja de ser el atributo psíquico de los sujetos para ser, más bien, el contexto de cada yo (321-322). Así pues, es en este darse en el momento actual o presente sin fisuras que se constituye el núcleo del desarrollo de la experiencia pura. Desde el punto de vista de los componentes de la conciencia, puede hablarse de un entramado complejo; no hay que olvidar, no obstante, que este punto de vista es reflexivo, derivado de la inmediatez con la que las cosas se dan originariamente. Así pues, prevalece el presente en el que corre el flujo de la conciencia. Poco importa aquí que dicho presente se encuentre, por decirlo de algún modo, preso de una percepción particular; sus marcos se extienden de actos particulares, como pueden ser observar una flor, a toda una serie perceptiva, como captar una melodía. Es a esto lo que Nishida llama atención. ${ }^{5}$

5 "El foco de la conciencia es en todo momento el presente, y la esfera de la experiencia pura coincide con la esfera de la atención. Pero la esfera de la experiencia pura no está necesariamente limitada a un solo foco de atención" (Nishida, 1995: 44). 
El esfuerzo conceptual aquí descrito puede parecer insuficiente por no esclarecer la supuesta inmediatez de la conciencia. A lo más, pareciera que la inmediatez, la así llamada unidad entre la conciencia y sus elementos, viene más bien de un elemento extrínseco a ella: el tiempo. Considerando esta objeción, proveniente de las propuestas psicológicas que proceden mediante la diferenciación de facultades, Nishida propone pensar a la conciencia como una "inclinación unificadora" cuya principal característica se manifestaría en la integración de sus actos (1995: 46). Atento a la innegable temporalidad de la conciencia, Nishida no podía hacer más que incluir esta característica como un desarrollo que se encuentra animado por una voluntad de unificación, vigente y siempre actuante. Es por ello por lo que podemos leer:

Como toda entidad orgánica, un sistema de conciencia manifiesta su totalidad mediante el desarrollo ordenado y diferenciado de cierta realidad unificadora. Cuando una conciencia comienza a emerger, la acompaña una actividad unificadora en la forma de un sentimiento de inclinación. Esta actividad dirige nuestra atención y es inconsciente cuando la unidad es estricta o no está perturbada desde afuera. (Nishida, 1995: 46)

El carácter fundamental de la conciencia ${ }^{6}$ no está en el futuro, sino en el presente. La voluntad es entonces un acto por sí mismo, la actividad unificadora que anima a la conciencia. Sin embargo, no por ello hay que pensar en la experiencia pura como desprovista de significación, como un bloque homogéneo en el que no cabe distinción alguna. Una vez más, Nishida reconoce el entramado que compone el flujo de la conciencia. Ejemplo de ello es su exposición del acto del juicio: juzgar que algo es de un determinado color sólo puede hacerse con base en sensaciones del pasado. Los juicios son, en este sentido, relaciones de experiencias pasadas que, no obstante, no modifican en nada la experiencia pura (Nishida, 1995: 48). Lo que se debe retener aquí es, más bien, el presente en el que se dan las cosas, la modalidad en que "todo es actualidad" (Nishida, 1995: 46).

Mientras que las nociones de inmediatez y de unidad son privilegiadas por Nishida, Husserl parece cuestionarlas y abandonarlas para poder dar rienda suelta

6 Si bien el intercambio entre los conceptos de 'conciencia’ y 'experiencia pura' puede parecer arbitrario, Nishida lo autoriza (o al menos lo permite la traducción consultada): "Una experiencia verdaderamente pura no tiene significación alguna; es simplemente conciencia presente de hechos tales como ellos son” (1995: 44). 
a la fenomenología trascendental. ${ }^{7}$ Salvando las distancias entre ambos conceptos, ${ }^{8}$ la actitud natural que permea todo acto de la conciencia puede coincidir con algunas notas de la experiencia pura. Si el rasgo distintivo de la experiencia pura reposa en el presente en el que se dan cada uno de sus actos, ¿cómo no ver una similitud con la actitud natural, cuyo rasgo principal, tal y como Husserl explica en The Amsterdam Lectures (1997), está en la aceptación continua de la realidad, en el hecho de estar capturado por ella? "Percibir algo significa que nos ocupamos con el molino percibido, que nos enfocamos en él y solo en él"', dice Husserl, sugiriendo con ello que la dación habitual de las cosas se da en un flujo ininterrumpido en el que la distinción entre acto perceptivo y objeto no tiene cabida (217 [306], traducción propia). ${ }^{10}$ Es por esta razón por la que el yo y sus procesos son anónimos, esto es, no explícitos (Husserl, 1997: 217 [306]). Ahora bien, hay una cuestión que surge y que exige ser contestada, a saber, el problema trascendental, la relación entre conciencia y mundo (Husserl, 1997: 237 [330]), y es aquí en donde las diferencias entre Nishida y Husserl parecen multiplicarse inconteniblemente. Aunque el problema trascendental haya sido elaborado por Descartes y reformulado por la psicología empírica de Locke, la cuestión sigue abierta para Husserl. La razón de ello se debe a que dicho problema ha sido abordado únicamente desde perspectivas psicológicas (Husserl, 1997: 237 [330]). A los ojos de Husserl, esto es cuestionable por circular: al intentar resolver el problema trascendental a partir de la psicología, la mente es concebida como el atributo de un ego humano perteneciente a una espaciotemporalidad determinada y, por ende, al mundo (Husserl, 1997: 240 [333]). En otras palabras, el psicologismo responde al problema trascendental desde aquello que está en cuestión. El punto de partida ha de ser otro, y es aquí donde los conceptos de epojé y reducción, firma distintiva de la fenomenología trascendental, aparecen.

La epojé fenomenológica es un cambio de actitud introducido por Husserl para echar luz sobre el carácter constitutivo de la conciencia. En lugar de asumirla como indubitable o dudosa, la existencia del mundo pasa a tener un carácter fenoménico

7 No debe pensarse, sin embargo, que el único modo en que la fenomenología tematizó la conciencia fue reflexivo. Consciente de la discrepancia entre la reflexión y el contenido de conciencia que tematiza (San Martín, 2015: 88-99), Husserl se refirió a otros modos de conciencia, como el corporal (Behnke, 2016: 41-42).

8 Que la actitud natural y la experiencia pura sean conceptos equiparables, es una afirmación que necesita de un desarrollo más profundo que el ofrecido aquí. Por lo anterior, asumiré de manera hipotética su convergencia.

9 "Perceiving something means we are occupied with the perceived windmill; we are focused on it and only on it".

10 Los corchetes en las citas de Husserl se refieren a la paginación de Husserliana. 
como mero correlato intencional de la conciencia. ${ }^{11}$ Imposible no ver en este cambio de actitud un paralelismo con el intento de duda practicado por Descartes en sus meditaciones, en las que el curso del mundo se ve interrumpido con tal de descubrir una fuente última e indubitable para las creencias. A pesar de la herencia cartesiana, Husserl no tarda en aclarar que su esfuerzo tiende menos a dudar de la existencia de las cosas que se le presentan a la conciencia, que a practicar una especie de desconexión del curso de su presentación (Husserl, 2013: 141[62]). El mundo de la actitud natural no es negado por el recurso de la duda; su existencia es mantenida a título de fenómeno con pretensión de validez dentro de la epojé (Husserl, 1973: 58-59). Al hacer esto, la fenomenología pretende conquistar un nuevo dominio: el de la conciencia o ego con sus múltiples cogitaciones (Husserl, 2013: 147 [67]). Por esta razón la epojé implica una reducción (reconducere), una reconducción al correlato entre conciencia y mundo. ${ }^{12}$ La distinción fundamental que hay que retener aquí es la de nóesis y nóema, acto de conciencia y lo percibido en cuanto tal. ${ }^{13} \mathrm{~A}$ ambos polos de la vida de conciencia subyace un polo egoico, centro que permanece idéntico a lo largo de los actos que irradian de él. Sólo por medio de este cambio de actitud puedo darme cuenta de que el yo, concebido trascendentalmente, tiene un rol constitutivo en lo que respecta al sentido del mundo. ${ }^{14}$

Es aquí en donde se halla un callejón sin salida. La unidad originaria de la experiencia que Nishida busca restituir en Indagación del bien parece ser lo que la epojé busca romper. Comprender el carácter constitutivo de la conciencia, tal y como Husserl intentó hacerlo, exigía poner entre paréntesis la inmediatez de las cosas, abrir una escisión en el seno de su espontaneidad con tal de poder discernir los actos por

11 "[Tras practicar la epojé] no sólo la naturaleza corporal sino la totalidad del concreto mundo circundante de la vida ya no es para mí, desde ahora, algo existente, sino sólo un fenómeno de ser" (Husserl, 1973: 56). En Amsterdam Lectures, el nuevo punto de partida se da a partir de un 'salto eidético' (Husserl, 1997: 240-241 [334]).

12 Retomo aquí una observación de Françoise Dastur, para quien la reducción es "un reconducir [reconducere], llevar de vuelta a la conciencia a su verdad que es ser conciencia constituyente" (Dastur, 2004: 94).

13 Esta distinción complica el cargo de dualismo. Irreductible al objeto real -por ejemplo, al árbol percibido-, el nóema es inmanente a la percepción sin por ello confundirse con los elementos ingredientes de la intencionalidad (Husserl, 2013: 293-296[202-205]). La nóesis o acto intencional remite a una historia que enraíza a la conciencia en el mundo. Para ver las implicaciones de lo anterior desde una perspectiva que enfatiza el aspecto genético de la conciencia, véase Derrida (1990).

14 "Por la 'èpokhê fenomenológica yo reduzco mi yo natural humano y mi vida psíquica [...] a mi yo fenomenológico-trascendental [...] El mundo objetivo [...] extrae [...] todo su sentido y su validez de ser $[\ldots]$ de mí mismo, de mí en cuanto yo trascendental" (Husserl, 1973: 66). 
los que aparecen. Por su parte, Nishida va en una dirección contraria toda vez que su propósito es el de capturar la unidad primigenia entre conciencia y mundo, entre flujo de la experiencia y momentos particulares. Considerando lo anterior, puede afirmarse que hay una diferencia insalvable entre ambos pensadores: los términos empleados por los dos no sólo son distintos, sino que también están moldeados por una metodología que los conduce por senderos opuestos. A continuación, intentaré poner a prueba esta conclusión con base en las observaciones que tanto Nishida como Husserl hacen sobre la intersubjetividad.

\section{El mundo y los otros: Nishida y Husserl de cara al problema de la intersubjetividad}

De acuerdo con Shigeru Taguchi (2019), en el primer periodo de su pensamiento "Nishida no toma muy en serio la diferencia entre yo y el otro" (105). Semejante interpretación adquiere sentido cuando se le contrasta con esta afirmación de Nishida: "la unidad de la conciencia en un individuo y la conexión de la conciencia de sí y del otro difieren solo en grado; no deberían ser distinguidas" (Nishida citado en Taguchi, 2019: 105). ${ }^{15}$ Es quizás esta razón la que lo llevó a elaborar una ontología capaz de integrar esta relación, así como la dinámica existente entre lo múltiple y lo uno. Curiosamente, la acusación de solipsismo lleva a Husserl a un sendero similar al emprendido por Nishida en lo que respecta a la constitución del mundo objetivo. Son las observaciones de "The Logic of Topos", escrito del filósofo japonés, y Meditaciones cartesianas las que quizás puedan tender un puente entre ambos planteamientos.

En Nishida Kitarō’s Chiasmatic Chorology, John W. Krummel (2015) encuentra una correspondencia entre las observaciones generales que Nishida hace sobre el lugar (topos) y su análisis del mundo sociohistórico (79). En efecto, Nishida parece confirmar esa identificación: "a menudo he comparado el absoluta y contradictoriamente topos autoidéntico, el mundo del presente absoluto, el espacio histórico con una esfera infinita" (Nishida, 1986: 25).${ }^{16}$ Ahora bien, tal identidad reposa en una

15 "The unity of consciousness in one individual and the connection of the consciousnesses of self and other differ only in degree and should not be discriminated absolutely."

16 "I have often compared the absolutely contradictorily self-identical topos, the world of the absolute present, the historical space to an infinite sphere". Quiero sugerir aquí que Nishida toma el término de "espacio histórico" como una determinación del "absoluta y contradictoriamente topos idéntico a sî" (Nishida, 1986: 25). Para más al respecto, véase Maraldo (2017: 92-93). 
lógica contradictoria, dinámica en la que la identidad de las cosas depende de su oposición o, dicho en términos nishidianos, negación mutua (Nishida, 1986: 3). ${ }^{17}$ Importa resaltar aquí que dicho proceso no es unidireccional, como si Nishida buscara privilegiar lo que uno de los términos, llámese conciencia o sujeto, puede hacer frente a aquello que lo niega. Por el contrario, la lógica contradictoria implica que los términos en cuestión, ya sea la conciencia o el mundo, influyen uno en el otro en la oposición. Es por esta razón que Nishida, al pensar el mundo a partir de la lógica contradictoria, puede afirmar que "al contener la autonegación dentro de sí y reflejándose a sí dentro de sí, el mundo sigue formándose infinitamente a través de la negación de la negación" (Nishida, 1986: 4). ${ }^{18}$ La negación recíproca entre las cosas no es, por lo tanto, el resultado de una acción sobre un objeto inerte o pasivo; se trata más bien de una acción bidireccional cuyo resultado conduce a la formación de los términos opuestos, no a su anulación. Las cosas se oponen y, al hacerlo, se reflejan y se afirman.

Podría parecer que Nishida habla de una oposición binaria en la que se encontraría, por un lado, el mundo y, por el otro, el yo, término con el que se agruparían todos los individuos sin considerar sus diferencias. No obstante, los yoes son pensados en "The Logic of Topos" como mundos, lo que lleva a considerar el rol de la multiplicidad en la dinámica contradictoria ya descrita. Primero, ¿qué subyace a la idea del yo como mundo? Se trata del hecho de que el mundo es apropiado subjetivamente por el yo. ${ }^{19}$ Pero la relación no se estanca allí; el yo se vuelve el locus de la autoformación [self-formation] del mundo, uno de los tantos lugares desde los cuales se expresa y se constituye su ser. Tal planteamiento puede extenderse a la intersubjetividad. A pesar de reconocer, según Krummel (2015), una separación y una discontinuidad radical entre yo y otro, Nishida (1986: 81) también sugiere que el sentido de nuestro yo está moldeado por otras personas. Todavía más, la autoconciencia [self-consciousness] no surge "within a simply closed-off self; it arises only when the self transcends itself and

17 "That two things maintain their uniqueness as they stand opposed to each other and negate each other means that they are mutually conjoined and compose one form." (Nishida, 1986: 3)

18 "[c]ontaining self-negation within itself and reflecting itself within itself, the world continues to form itself endlessly through the negation of negation, i. e., self-affirmation."

19 "Que actuemos conscientemente significa que cada uno de nosotros, un punto expresivo del mundo, forma el mundo expresándolo en nuestro yo: el mundo es subjetivamente apropiado en nuestro yo." "That we act consciously means that each of us - an expressive point of the world-forms the world by expressing it in our self: the world is subjectively appropriated by our self.') (Nishida, 1986: 5; las cursivas son mías). 
faces the other" (Nishida, 1986: 5-6). El desarrollo del mundo, incluyendo a los yoes en él, se da entonces no sólo de manera interpersonal, sino también en sociedad; el yo, su identidad, se refleja en el otro y se difracta en él, dando como resultado una multiplicidad de ángulos desde los cuales el mundo es percibido:

En el mundo de la identidad absolutamente contradictoria las individualidades multiples, en cuanto perspectivas, poseen el carácter de un mundo. [...] El mundo, en cuanto identidad absolutamente contradictoria, en cuanto autodeterminación del presente absolute, proyectándose dentro del yo y actuando a partir de esta perspectiva dinámica, se va formando a sí mismo. El mundo posee aquí por su propio orden. Nuestros 'yoes', en cuanto multiples individualidades de este mundo, en cuanto perspectivas de este mundo, a la vez que expresan el mundo en sí mismos, adquieren una direccionalidad por su participación en la direccionalidad de la autoformación del mundo. (Nishida, 2006: 29-30) ${ }^{20}$

La lógica de los contrarios echa luz sobre la coincidencia entre los puntos focales del mundo. Si los individuos, además de reflejar al mundo, también se oponen entre sí, sus experiencias, sus vivencias, todo lo que forma parte de ellos se difractará en el otro, de tal manera que la coincidencia entre su aprehensión del mundo se vuelve posible. Cuestión aparte es la posibilidad del desacuerdo o el consenso entre estas perspectivas.

El reconocimiento de la alteridad radical del otro y la aprehensión múltiple del mundo son las resonancias que encuentro con los planteamientos de Husserl sobre la intersubjetividad, cuya elaboración es, al menos en Meditaciones cartesianas, indisociable de la respuesta de la fenomenología frente a las acusaciones de solipsismo. El problema del solipsismo surge debido a que la epojé "encierra" al meditante en su propia corriente de vivencias. Ello no significa, sin embargo, que la fenomenología esté desprovista de herramientas que le permitan pensar la cuestión de la alteridad, y es a este título que la exposición debe desarrollarse teniendo al otro como contenido noemático. Por ende, es necesario que la descripción pase por los modos en los que la otredad se configura, se aclara y se descubre dentro de la vida intencional de la conciencia, sin por ello dejar de prestar atención a que el otro se presenta como un alter-ego igualmente perceptor y constituyente del mundo (Husserl, 1973: 152-154).

20 Debido a que ésta es una cita larga, preferí retomar la traducción que Juan Masiá y Juan Haidar hicieron de "The Logic of Topos". 
Dándose a la tarea de describir estos procesos, Husserl practica una 'epojé temática' que suspende momentáneamente toda referencia a los seres del género del yo y, por ende, a toda comunidad (1973: 155). Tal movimiento pone al descubierto la función del cuerpo como sustrato de todas las posibilidades de percepción (Husserl, 1973: 155). Es esto lo que permite hacer el tránsito a la experiencia de la alteridad, la cual se desenvuelve, retomando los términos de Husserl, como una 'filiación de sentido': cuando el otro se presenta ante mí, entra a mi campo perceptivo un cuerpo análogo al mío. Se realiza entonces una transferencia de índole "aperceptiva", pues el cuerpo físico del otro me indica, por medio de la similitud que hay entre su cuerpo y el mío, que hay otro yo (alter ego) frente a mí. A diferencia de la experiencia que tengo de cualquier otro cuerpo físico, la percepción del otro como alter ego (de aquí en adelante, la apresentación analógica) me implica a mí mismo en cuanto cuerpo orgánico similar al de aquel que se me aparece. ${ }^{21}$

A pesar del abismo que se tiende entre el yo y el otro, la apresentación analógica cuenta con sus propias modalidades de verificación, y es aquí donde aparece la espacialidad relativa a los cuerpos. Tanto a mi cuerpo como al cuerpo del otro corresponden dos modos de espacialidad: se trata de mi aquí central y del "allî" al que me remite la aparición del otro. En lugar de entender ambas referencias como puntos fijos en el espacio, hay que ver en el aquí y en el allí dos localidades que se desplazan a la par que las conciencias encarnadas (Husserl, 1973: 184). Gracias a dicha posibilidad puedo imaginarme como estando en el lugar del otro, lo que repercute en la constitución del mundo objetivo toda vez que me puedo representar como percibiendo el mismo objeto desde una perspectiva distinta. Por más infranqueable que sea el

21 Lo anterior necesita de varias precisiones. La idea de una aparición del otro es, tal y como Didier Franck indica (2014), engañosa. Tomada literalmente, sólo puede aceptarse si nos restringimos al campo perceptivo actual, lo cual es imposible toda vez que la percepción consta de horizontes que necesariamente implican al otro (Franck, 2014: 105). En segundo lugar, la filiación de sentido aquí descrita implica una síntesis pasiva. Con lo anterior, Husserl se refiere al hecho de que toda percepción remite a una fundación originaria [archi-foundation] en virtud de la cual los objetos nuevos son reconocidos como familiares o extraños por la conciencia (Franck, 2014: 108). La apresentación analógica significa así que la conciencia confiere el sentido egoico al cuerpo extraño a través de "una analogía sensible o una similitud que, pasivamente, afecta al ego" ("a sensible analogy or similitude that passively affects the ego") (Franck, 2014: 108). ¿Qué analogía sensible? La similitud entre su cuerpo y el mío. A diferencia de Taguchi, las observaciones de Franck respetan el carácter encarnado de la conciencia a partir de la percepción por escorzos (2014: 38-39). Creo posible aventurar que llega a una conclusión similar a la de Taguchi - Husserl afirma que el otro se encuentra irreductiblemente en la conciencia-, pero por otros medios conceptuales - la síntesis pasiva y el horizonte de percepciones. 
abismo entre yo y el otro, hay cierta identidad que se establece a nivel corporal. El allí del otro es, en tanto ego, un aquí que me remite inmediatamente a mí mismo como ocupando su lugar:

El hecho de que mi cuerpo físico orgánico sea aprehendido y sea aprehensible como cualquier otro cuerpo físico natural que existe y se mueve en el espacio, está manifiestamente ligado a la posibilidad que se enuncia en las palabras: yo puedo cambiar mi posición de tal manera que podría transformar todo allí en un aquí, es decir, que yo podría ocupar corporalmente cualquier lugar espacial, mediante la libre modificación de mis cinestesias, y en particular del andar. (Husserl, 1973: 184)

Así pues, la alteridad permite comprender la constitución del mundo como un acto realizado por distintos yoes cuyas perspectivas se complementan.

Considerando lo anterior, ¿no puede decirse que Nishida y Husserl coinciden al menos en este punto? El privilegio de la descripción en primera persona dentro de la fenomenología parece obstaculizar la convergencia entre los dos. Al describirnos la dinámica de los contrarios, Nishida insiste en que la interacción de las cosas del mundo es multidireccional: al negarse, los opuestos se reflejan y difractan entre sí, arrastrando en este proceso las vivencias que llevan consigo; no habría, por lo tanto, un punto de vista privilegiado desde el cual trazar esta lógica. Sin embargo, el lugar desde el cual habla Husserl responde a un hecho tan innegable como necesario, a saber, no hay acceso al otro por cuanto es yo, fuente constitutiva de sentido. Por lo anterior, se vuelve necesario reconocer el límite de la descripción de su dación sin por ello renunciar a su realización. Es en esta tensión entre opacidad y visibilidad que se tiende la convergencia. El otro expresa una parte del mundo, una parte que puede estar en contradicción o en concordancia con la mía. Como sea, la posibilidad del mundo -entendiéndolo no como un objeto previamente dado, sino como un horizonte en devenir - depende de esta disimetría absoluta entre el yo y el otro, de la transponibilidad de perspectivas y de su posible armonización.

\section{Conclusiones}

En las páginas precedentes intenté dar razón de dos cosas. Primero me interesé en la divergencia entre los planteamientos de Nishida y Husserl, aspecto que encontra- 
ría su raíz en el método que anima las observaciones de los dos. La fenomenología trascendental y la experiencia pura parecen ir en direcciones opuestas: la primera busca romper la inmediatez de la experiencia, cosa que los esfuerzos de Nishida en Indagación del bien parecen restituir. Si bien mencioné que la similitud entre la experiencia pura y la actitud natural es asumida de manera hipotética, el hecho de que Nishida se oponga al proceder reflexivo, propio de la fenomenología, habla a favor de esta tesis. Ahora bien, la divergencia deja de ser tan radical al considerar las observaciones que Nishida y Husserl hacen de la intersubjetividad. Es cierto que ninguno de los dos autores sigue la misma ruta para abordar esta cuestión; no obstante, tanto Nishida como Husserl elaboran un concepto de alteridad en cuyo correlato se inscribe la intersubjetividad y, por ende, la objetividad del mundo. Con tal de proseguir la discusión, aquí pueden plantearse dos rutas preliminares.

Hay un aspecto que no consideré directamente en este ensayo: la imbricación entre autoconciencia y alteridad. Mientras que éste parece ser un aspecto decisivo en "The Logic of Topos", Husserl parece asentar algunas líneas que van hacia esa dirección sin realmente posicionarse - o al menos no lo hace en el contexto de Meditaciones (Taguchi, 2019: 102-104)—. Quien sí le dará un papel constitutivo a la alteridad en la experiencia de sí es Derrida (2000), y el ejemplo más elocuente de ello está en Le toucher: la experiencia del tocarse será interpretada como anunciando una alteridad, afirmándose así su irreductibilidad a la mera aprehensión de sí mismo (200). ${ }^{22}$ Es importante añadir que la otredad de la que habla Derrida no es sólo la del alter-ego, sino también la de la cosa extensa cuya mostración se realiza parcialmente. En otras palabras, su concepto es más general. La relación entre lo otro y el yo, así como el peso constitutivo de la alteridad en la relación consigo mismo, exige la consideración de una alteridad radical no encarnada en un yo.

La viabilidad de mi intuición depende aquí de otro elemento no menos importante, a saber, lo que los conceptos de estos pensadores mientan. Tal y como sugiere David W. Johnson (2020), los conceptos filosóficos no pueden abstraerse del lenguaje al que pertenecen, y cualquier trabajo de filosofía comparada exige tener en la mira esta dificultad (386). Con ello no quiero implicar que los planteamientos de Nishida

22 Un movimiento similar se encontraría ya en La voix et le phénomène (1967), obra en la que Derrida muestra como la experiencia del escuchar-se no ocurre en un presente pleno, sino en una temporalidad que precisa de la retención y, por consiguiente, de la mediación (98-102). Lo anterior haría de esta experiencia una hetero-afección, una experiencia en donde la otredad se cuela en la intimidad de la conciencia. 
o de Husserl (o incluso los de Derrida) sean tan diferentes que cualquier tentativa de conciliación ha de fallar; la idea de Johnson debería conducir, más bien, al descubrimiento de las singularidades propias de cada uno de estos planteamientos, aspecto que parece ser olvidado cada vez que se intenta poner en diálogo a pensadores de horizontes distintos. Sólo en lo que un pensador advierte de manera distinta puede construirse una verdadera discusión; de otro modo, rumiamos en lo que puede ser asimilable a nuestros conceptos.

\section{Referencias bibliográficas}

BeHnKe, Elizabeth. (2016). "La vida operativa: The transcendental disclosure of operatively functioning life”. Acta Mexicana de Fenomenología, (1), 29-48.

DASTUR, Françoise. (2004). "Réduction et intersubjectivité". En Françoise Dastur, La phénoménologie en questions : langage, altérité, temporalité, finitude (pp. 83-99). Libraire Philosophique J. Vrin.

DERRIDA, Jacques. (1967). La voix et le phénomène: Introduction au problème du signe dans la phénoménologie de Husserl. Presses Universitaires de France.

DerRIDA, Jacques. (1990). Le problème de la genèse dans la philosophie de Husserl. Presses Universitaires de France.

DerridA, Jacques. (2000). Le toucher, Jean-Luc Nancy. Éditions Galilée.

Franck, Didier. (2014). Flesh and Body: On the Phenomenology of Husserl (Joseph Rivera y Scott Davidson, Trads.). Bloomsbury.

Husserl, Edmund. (1973). Meditaciones cartesianas (Mario. A. Presas, Trad.). Ediciones Paulinas.

Husserl, Edmund. (1997). The Amsterdam Lectures. En Thomas Sheehan y Richard E. Palmer (comps. y trads.), Psychological and Transcendental Phenomenology and The Confrontation with Heidegger (1927-1931) (pp. 213-253). Springer.

HUSSERL, Edmund. (2013). Ideas relativas a una fenomenología pura y una filosofía fenomenológica. Libro Primero: Introducción general a la fenomenología pura (Antonio Zirión Quijano, Trad.,). Fondo de Cultura Económica. 
Johnson, David W. (2020). "The Limits of Language: Philosophical Hermeneutics and the Task of Comparative Philosophy". Journal of Speculative Philosophy, 34(4), 378389. https://doi.org/10.5325/jspecphil.34.3.0378

Krummel, John W. M. (2015). Nishida Kitaro's Chiasmatic Chorology: Place of Dialectic, Dialectic of Place. Indiana University Press.

Maraldo, John C. (2017a). "La ontología de la historia de Nishida Kitarō". En Rebeca Maldonado (Comp.), Tránsito(s) y resistencia(s): ontologías de la historia (pp. 63101). Editorial Itaca.

Maraldo, John C. (2017b). "What Phenomenologists Can Learn from Nishida about SelfAwareness". En John C. Maraldo, Japanese Philosophy in the Making I: Crossing Paths with Nishida (pp. 299-349). Chisokudo.

NishidA, Kitarō. (1986). "The Logic of 'Topos' and the Religious Worldview, Part I" (Yusa Michiko, Trad.). The Eastern Buddhist, 19(2), 1-29.

Nishida, Kitarō. (1995). Indagación del bien (Alberto Luis Bixio, Trad.). Gedisa.

Nishida, Kitarō. (2006). Pensar desde la nada: ensayos de filosofía oriental (Juan Masiá y Juan Haidar, Trads.). Ediciones Sígueme.

SAN MARTín, Javier. (2015). La nueva imagen de Husserl. Lecciones de Guanajuato. Editorial Trotta.

Stevens, Bernard. (2008). Invitación a la filosofía japonesa. Bellaterra.

TaguchI, Shigeru. (2019). "Consciousness Without Boundaries? The Riddle of Alterity in Husserl and Nishida". En Shigeru Taguchi y Andrea Altobrando (Comps.), Tetsugaku Companion to Phenomenology and Japanese Philosophy (pp. 97-110). Springer. 